\title{
PREVALENCIA DE DIABETES MELLITUS EN LA CIUDAD DE PASTO, NARIÑO COLOMBIA
}

\author{
Miguel Angel Suárez Russi ', Clara López de Mesa Melo ${ }^{2}$, Fabio Urbano Buchelli ${ }^{3}$, \\ Armando Coral Burgos ${ }^{2}$, Edgar Villota Ortega ${ }^{2}$, Harlem Poveda de Ruiz ${ }^{1}$ y Leobardo Suarez Russi ${ }^{2}$
}

Se realizó un estudio de tipo descriptivo, transversal, en la población adulta, mayor de 20 años, hombres y mujeres, habitantes de la zona urbana de la ciudad de Pasto, localizada en la zona suroccidental de Colombia, sus características son similares en alimentación y hábitos culturales al mayor porcentaje de los habitantes de Colombia de las zonas montañosas andinas, con una altura sobre el nivel del mar de 2.650 metros y una temperatura promedio de 11 grados centígrados.

De una población adulta mayor de 20 años se seleccionó una muestra aleatoria sistemática de 1.430 personas. Aceptaron participar en forma voluntaria, aprovechando la estratificación de salud que tiene este municipio, distribuídos en seis sectores, sin una delimitación clara de clases sociales. Se realizó una encuesta domiciliaria sobre antecedentes familiares de diabetes y obesidad. Además se evaluaron otras variables como peso, talla, índice de masa corporal, escolaridad, para posteriormente citarlos y realizar una prueba de tolerancia a los carbohidratos, con una carga estándar de 75 gramos de glucosa, en 720 personas. Se determinó la glicemia mediante técnica de glucosa oxidasa en un equipo semiautomático cuantificando la pre y postcarga. Se emplearón para la evaluación los criterios de Ia OMS y National Diabetes Data Group.

Al 6,0\% de la población se le detectó diabetes mellitus, distribuidos en el $3,6 \%$ para mujeres y el $2,4 \%$ para los varones, siendo mayor la proporción de diabéticos entre la cuarta y quinta década. El $54,6 \%$ de los diabéticos tenían un IMC superior a 25 , mientras que el 6,8\% tenían problemas de obesidad con IMC superior a 30 .

Hubo intolerancia a los carbohidratos en el $5,3 \%$ de la población cuando se evaluó este factor aceptando la recomendación mundial de valores de glicemia entre 7,8 $\mathrm{mmol}$ y $11,1 \mathrm{mmol}$ de glucosa en sangre venosa.

El $22,5 \%$ de los sujetos diabéticos tenía antecedente materno de diabetes y paterno del $9,8 \%$, siendo estadísticamente significativas estas cifras $(p<0.05)$. En los abuelos, el $11.1 \%$ de las abuelas maternas tenía diabetes mellitus. El antecedente familiar de obesidad más frecuente fue en la madre y en la abuela materna en el $26 \%$; en el $17 \%$ para el padre e igual para el abuelo materno.

El $44,7 \%$ de los diabéticos detectados se conocían diabéticos y el $31,3 \%$ estaba recibiendo medicación para su problema metabólico.

1 Universidad Nacional de Colombia. Facultad de Medicina.

2 Unidad de Endocrinologia Clinica San Pedro Claver. ISS Cundinamarca

3 Secretaria de Salud de Pasto, Nariño. 


\section{INTRODUCCION}

La diabetes mellitus es una enfermedad tan antigua como el hombre. Ya Aretaios de Capadocia en el siglo II la definía: "La diabetes es una enfermedad extraña que no se manifiesta muy a menudo entre los humanos. La carne y los huesos se diluyen en la orina; la humedad y el frio son su causa, como la hidropesía. Pero el líquido recorre el camino normal por los riñones y es excretado por la vejiga. Los enfermos no dejan de eliminar agua, de tal manera parece salir como de grifos abiertos...". (1)

La diabetes es una entidad que ataca a un gran número de personas de todas las áreas del mundo, sin distinción de condiciones sociales. sinembargo ha ido aumentando en forma progresiva, merced a la mayor longevidad de la población, a la mejoría de condiciones de vida y a la facilidad para detectar la enfermedad. $(1-3)$

La OMS define la diabetes mellitus como una entidad con aumento crónico de la glicemia, acompañado de síntomas, como sed intensa, micción profusa, pérdida de peso y estupor. Culminan en coma y muerte cuando no se administra un tratamiento efectivo. (3).

No siempre los síntomas de la diabetes son tan evidentes que permitan detectar la enfermedad en una fase temprana, razón por la cual se han creado algunas pruebas de laboratorio clínico, para evidenciar la alteración metabólica de los carbohidratos. Además es empleado como monitor de la enfermedad, aunque sólo detecta un instante de la glicemia. En la actualidad se han creado sistemas enzimáticos, de fácil ejecución, de gran rapidez, confiables y que permiten determinar los niveles de glicemia circulante (1-7).

La diabetes mellitus es considerada un factor de riesgo mayor $(1,3,4,8,9)$. Su prevalencia es variable dependiendo del tipo de población analizada. En el caso de los habitantes nicronesianos de la isla Nauru (10) la prevalencia de diabetes asciende a un $30 \%$ en población mayor de 20 años, en contraste con los polinesios, en donde sólo alcanza a un $2,7 \%$ en el mismo grupo de población. En los reportes de Suramérica, las cifras son menos variables, como en Venezuela es del $7 \%$, Uruguay el $6,9 \%$, Panamá el $2,5 \%$, en Cuba es del $8 \%(10,11)$. En Colombia los distintos estudios muestran cifras que oscilan entre el $2,5 \%$ y el $8 \%$ según los grupos estudiados (12-14) valores éstos intermedios dentro de las tasas de prevalencia mundial de diabetes en adultos.

El objetivo del presente estudio fue evaluar la prevalencia de diabetes mellitus en mayores de 20 años en una población urbana en el suroccidente de Colombia. Se emplearón las recomendaciones del concenso de la OMS de 1985 y del National Diabetes Data Group. (3).

\section{MATERIAL Y METODOS}

Se realizó un estudio de tipo descriptivo, transversal, en la población adulta, mayor de 20 años, hombres y mujeres, habitantes de la zona urbana de la ciudad de Pasto, localizada en la zona suroccidental de Colombia Sus características similares en alimentación y hábitos culturales al mayor porcentaje de los habitantes de Colombia que habitan en las sierras o zonas elevadas de las montañas andinas, con una altura sobre el nivel del mar de 2.650 metros y una temperatura promedio de 11 grados centígrados.

Se seleccionó una muestra de 1430 personas, elegidas en forma aleatoria. Todos ellos aceptaron ingresar al estudio en forma voluntaria, cumpliendo con las normas del protocolo de Helsinki. Se tuvieron en cuenta las caracteristicas de estratificación de la ciudad, donde no se observan tan delimitadas geográficamente las diferentes clases sociales.

Todos los sujetos incluidos en la muestra fueron habitantes de la zona urbana de la ciudad, adscritos a uno de los 6 centros de salud con que cuenta el Sistema de Salud Municipal. Algunos de ellos también eran beneficiarios de sistemas de salud prepagada estatales o particulares.

Se practicó una selección de acuerdo a la distribución geográfica de la ciudad, teniendo en 
cuenta las areas de mayor densidad poblacional, mediante el empleo de un instrumento de tipo encuesta previamente codificada. Su proceso de diligenciamiento fue ejecutado, por personal entrenado,en los sitios de habitación de los sujetos incluidos en la muestra.

Se analizaron las variables edad, sexo, talla, peso, índice de masa corporal, escolaridad, estado civil, antecedentes familiares y personales de diabetes. Además de lo anterior, se cuantificaron bioquímicamente, mediante técnica de química líquida, los niveles circulantes de glucosa en ayunas y postcarga.

De esta submuestra se seleccionó en forma aleatoria un total de 720 personas $(50,3 \%)$, a quienes se les administró una carga estandar de 75 gramos de glucosa (Despak (R)), diluidos en $350 \mathrm{ml}$ de agua. Estos se administraron en forma de bolo, inmediatamente después de habérseles tomado la primera muestra de sangre en ayunas, para su determinación bioquímica. Los sujetos seleccionados estuvieron en reposo durante los 120 minutos previos a la segunda muestra de sangre para la nueva evaluación de la glicemia. De este grupo se descartaron los pacientes diabéticos previamente conocidos.

Todos los pacientes venían recibiendo una dieta corriente, con un porcentaje elevado de carbohidratos, habitual en la zona.

Posterior a la realización de la encuesta los sujetos fueron citados en el respectivo centro de salud, para una toma de sangre con un período de ayuno no inferior a doce horas, empleando para su recolección un sistema al vacío, desechable. La muestra de sangre fue centrifugada y procesada subsiguientemente, en un equipo semiautomático marca "Labt system (R)", con sistema de glucosa peroxidasa de la casa Boheringer Manhein (R).

De la población total de 254.000 personas de la zona urbana de la ciudad de Pasto, 117.100 habitantes $(46,1 \%)$ fueron adultos mayores de 20 años. De estos se seleccionaron 1.430 suje- tos para ser incluidos en la muestra a estudiar, 0 sea el $1,22 \%$ de la muestra.

El criterio para denominar un sujeto diabético es el mismo utilizado por la OMS y el National Diabetes Data Group $(3,20)$. Es decir, aquel individuo cuya glicemia en ayunas o postprandial sobrepase los $200 \mathrm{mg} / \mathrm{dl}$ teniendo en cuenta que se trató de determinaciones realizadas en un solo día.

Métodos estadísticos: la información se procesó en computador mediante programa estadístico SPSSPC/t. Una vez determinada la prevalencia de la diabetes mellitus, se procedió a realizar una descripción de las caracteristicas de los pacientes diabéticos empleando las variables propuestas, para comparar proporcionalmente los hallazgos del grupo de diabéticos con el resto de la población estudiada (no diabéticos). Se utilizarón para este procedimiento pruebas estadísticas: Chi-cuadrado para proporciones, Mann-Whytney para variables no paramétricas, prueba de los signos para variables dicotómicas, análisis de varianza entre grupos (Oneway), distribución normal.

Las bases porcentuales en las distribuciones de frecuencia ( $100 \%$ ) se tomaron con los sujetos que contestaron con certeza. Es decir, no fueron incluidas las respuestas dudosas, como en el caso de las preguntas sobre los antecedentes familiares, en las cuales se incluyó la posibilidad de respuesta "no sabe".

Se consideró estadisticamente significativo $p<0,05$.

\section{RESULTADOS}

De acuerdo a los criterios aceptados por la OMS, encontramos que en la población mayor de 20 años, la prevalencia de diabetes mellitus fue del $6,5 \%(n=47)$, siendo de $3,6 \%$ para las mujeres y de $2,4 \%$ para los varones, con una proporción mayor en mujeres $(80,9 \%, n=38)$ y en hombres $(19,1 \%, n=9)$. La distribución de los diabéticos por grupos etáreos, fue más frecuente entre la cuarta y la quinta década de la vida (Figuras 1-4). 
Figura 1. PREVALENCIA DE DIABETES EN LA CIUDAD PASTO, NARIÑO

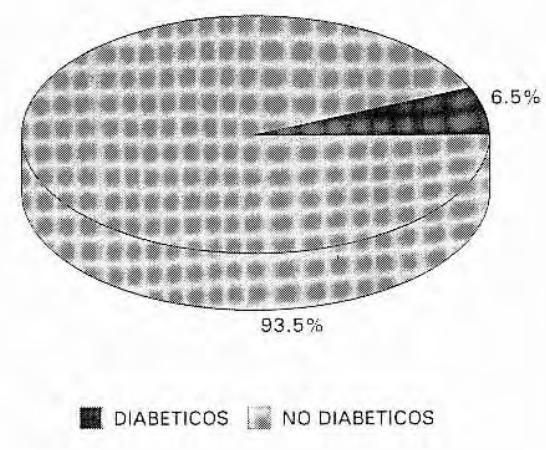

$N=1430$

Figura 2. PREVALENCIA DE DIABETES POR SEXO EN LA CIUDAD DE PASTO, NARIÑO

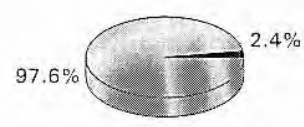
HOMBRES
$\mathrm{N}=373$

DIABETICOS $\square$ NO DIABETICOS

Figura 3. DISTRIBUCION PORCENTUAL DE PACIENTES DIABETICOS POR SEXO

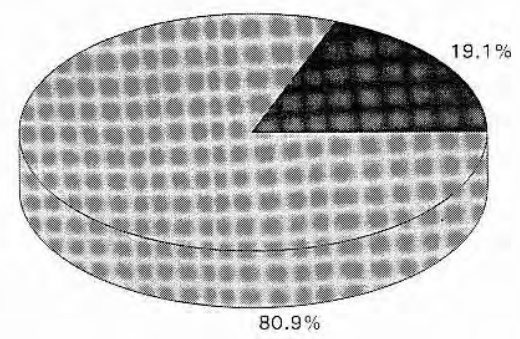

$N=47\left\langle 100^{4}\right.$ i
Figura 4. DISTRIBUCION PORCENTUAL DE PACIENTES

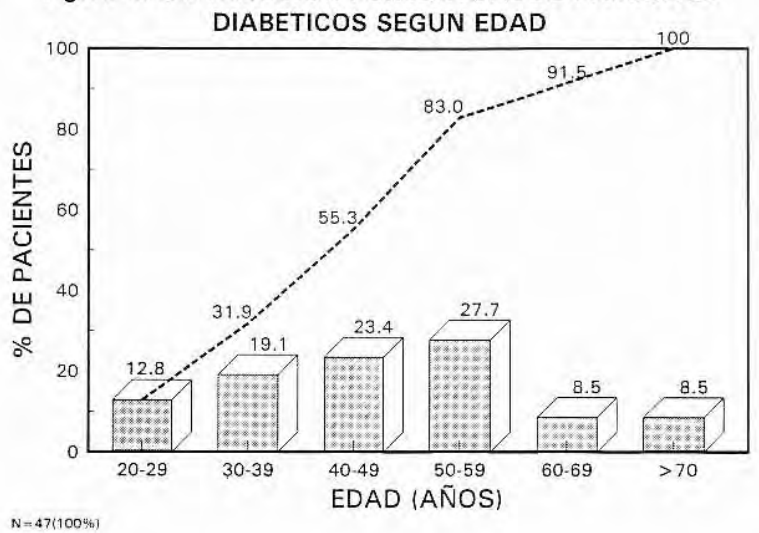

Las características sociales del grupo de pacientes diabéticos, mostró que el 59,6\% ( $n=28)$ eran personas instruidas con la educación básica y un $4.3 \%(n=2)$ eran analfabetas. El $74,5 \%$ $(n=36)$ fueron personas casadas o con uniones estables y un $25,5 \%(n=12)$ solteros.

El 54,6\% $(n=24)$ de los diabéticos estudiados tenía problemas de sobrepeso, Considerando un índice de masa corporal superior a 25 y un $6,8 \%(n=3)$ tenían problemas de obesidad con indice de masa corporal mayor de 30. (Figura 5)

Hubo intolerancia a los carbohidratos en el $5,3 \%$ de la población cuando se evaluó este factor aceptando la recomendación mundial de valores de glicemia entre $7,8 \mathrm{mmol}$ y $11,1 \mathrm{mmol}$ de glucosa en sangre venosa. (Figura 6)

Antecedentes familiares de diabetes: el 22,5\% de los sujetos diabéticos tenía antecedente materno de diabetes y paterno del $9,8 \%$, siendo estadísticamente significativas estas cifras $(p<0,05)$. En el caso de los abuelos, el $11,1 \%$ de las abuelas maternas fueron reportadas como positivas para diabetes mellitus. En los familiares restantes no fueron muy importantes los antecedentes. (Figura 7)

Antecedentes familiares de obesidad en los diabéticos: este antecedente fue más frecuente en el caso de la madre y de la abuela materna, con valores positivos en un $26 \%$, en tanto que la misma evaluación solo fue positiva en un $17 \%$ cuando se evaluó al padre y al abuelo materno, como se observa en la Figura 8. 
Figura 5. DISTRIBUCION PORCENTUAL DE PACIENTES DIABETICOS SEGUN OBESIDAD

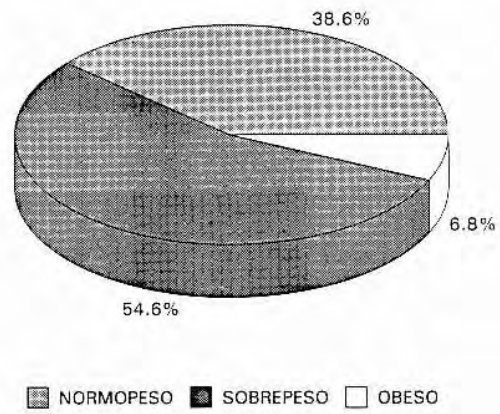

Figura 6. DISTRIBUCION PORCENTUAL DE SUJETOS CON INTOLERANCIA A LA GLUCOSA (140-199 mg/dl)

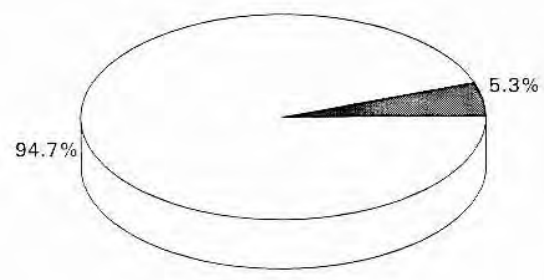

INTOLERANCIA

$N=1430 / 100 \%$

$\square \mathrm{sI} \square$ No

Antecedentes personales: el $44,7 \%$ de los diabéticos detectados se conocian diabéticos y el $31,3 \%$ estaba recibiendo medicación para su problema metabólico.

\section{DISCUSION}

Es bien conocido, que la incidencia de diabetes mellitus en población adulta, se ha venido evidenciando con mayor precisión en los últimos años, identificando a su vez que los sujetos de mayor edad son mas susceptibles a este tipo de entidad. Es reconocido que los aspectos genéticos tienen especial importancia, pues la concordancia observada en los gemelos diabéticos monocigóticos del Tipo II, llega a ser del ciento por ciento y la tasa de prevalencia acumulativa
Figura 7. FRECUENCIA DE ANTECEDENTES DE FAMILIARES DIABETICOS EN PACIENTES DIABETICOS

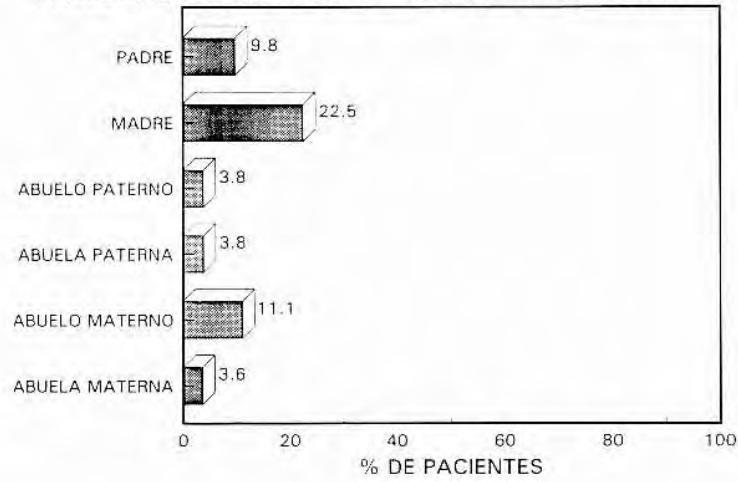

Figura 8. FRECUENCIA DE ANTECEDENTES DE FAMILARES OBESOS EN PACIENTES DIABETICOS

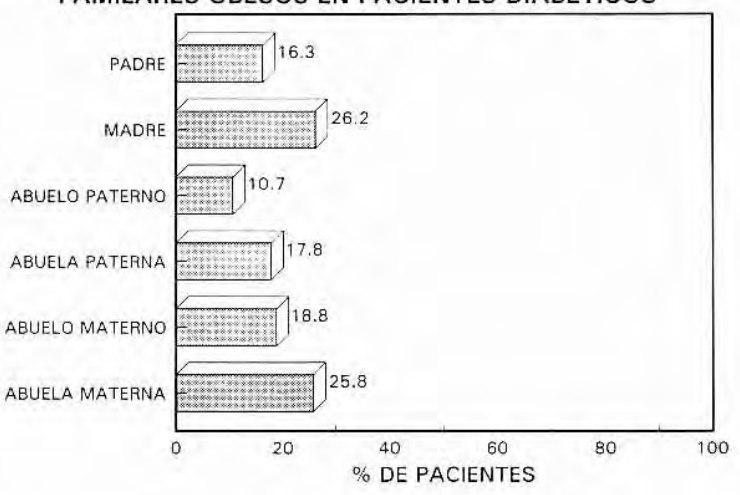

en los descendientes de ambos padres diabéticos se mantiene en rangos entre el 40 y $60 \%$; mientras que en los gemelos diabéticos Tipo I, solo es del $50 \%$ (15).

La prevalencia de diabetes mellitus encontrada en el presente estudio, fue del $6.5 \%$ en población mayor de 20 años. Esto muestra que la diabetes es una entidad cada vez con mayor repercusión dentro de los factores de riesgo cardiovascular en la población general, debido al incremento en el número de personas que la padecen.

En cuanto a los antecedentes familiares maternos de diabetes, fueron positivos en un $22,5 \%$, dato similar a los reportados por el 
estudio cubano (11); en tanto que los antecedentes diabéticos de los padres fueron sólo de un $9,8 \%$, cifras estadísticamente significativas $(p<0,05)$, al compararlas con las obtenidas proporcionalmente con el resto de sujetos no diabéticos estudiados en el presente estudio.

Un dato interesante se observó al hacer la evaluación de sujetos intolerantes a la glucosa, su proporción llegó al ser del 5,3\%, obervándose un sobrepeso importante en todos ellos, resaltando el concepto ya conocido en la literatura médica de la asociación de la obesidad y la resistencia insulínica. $(16,17)$.

Es conocido que la retinopatía y la nefropatía diabética son alteraciones altamente específicas del sistema microvascular del diabético, cuyo riesgo de lesión depende fundamentalmente del nivel de hiperglicemia y de la duración de la misma $(18,19)$. Por esta razón resulta particularmente importante hacer el diagnóstico de diabetes mellitus, a una edad en la cual se puedan adelantar campañas educativas tanto para el diabético como para su familia. Así por lo menos se le retarda la aparición de las complicaciones neurológicas y microvasculares, pues hasta el momento no se ha podido demostrar que los tratamientos, por muy estrictos que sean,lleguen a impedir que este tipo de alteraciones se presente, Lamentablemente cuando lo hacen, originan costos muy elevados para el paciente y para la sociedad, situación preocupante para una colectividad que no dispone de recursos adecuados para los servicios de salud.

La detección de la diabetes ha demostrado incluso en los países con mejores condiciones económicas que solamente se conoce el $50 \%$, o con diagnóstico previo, en tanto que la otra mitad pasan inadvertidos. Por esta razón se ha pretendido establecer esquemas en donde se pueda demostrar que la lenta desaparición de la glucosa y la hiperinsulinemia, puedan ser empleados como elemento predictor de desencadenamiento de la diabetes, pues tampoco el empleo de la proteínas glicosiladas ha resultado útil en este proceso. (20-22)

Diferentes autores (23-25), han mostrado la fuerte asociación entre la progresión de la obesi- dad y la alteración observada en la curva de tolerancia a la glucosa, o la diabetes franca, sin observar que la pérdida de peso se regrese a las condiciones normales de tolerancia a la glucosa. Esta razón obliga a ser prudentes en la evalución de diabéticos obesos. En el presente estudio encontramos que el $61,4 \%$ de los diabéticos eran obesos, con una diferencia estadísticamente significativa al comparar estos valores con la población no diabética $(p=0,01)$, datos estos similares a los reportados en la literatura. (23).

Los antecedentes maternos de obesidad fueron del $26,2 \%$ y de $16,3 \%$ en el padre, en tanto que los abuelos tenían antecedentes de obesidad en el $18 \%$ se evidencia de esta forma que quizá el fenómeno de sobrepeso esté dado en parte por características genéticas, o por condiciones culturales y nutricionales que no siempre muestran una diferencia estadísticamente significativa cuando se comparan con el grupo de población general.

\section{SUMMARY}

A descriptive, transversal study of an adult population - men and women over 20 years of age - was carried out in the urban area of Pasto (altitude $2,650 \mathrm{~m}$ above sea level $/ 11^{\circ} \mathrm{C}$ ) a city located in the southwest of Colombia, having similar features regarding nutrition and cultural habits to those of the inhabitants of the Andean area.

A systematic aleatory sample of 1,430 volunteers was taken from a diabetic and obese adult population determined by questionnaire which took into account such variables as height, weight, body mass index and educational level. From the results, appointments were made for carbohydrate tolerance tests; a standard of $75 \mathrm{~g}$ of glucose being established in 720 people. Glycemia was determined by oxidized glucose technique using a semiautomatic equipment quantified pre and post loading. This evaluation utilized WHO and National Diabetes group criteria. $6.5 \%$ of the sample were found to have diabetes mellitus $(3.6 \%$ women and $2.4 \%$ men with a high proportion in their 30 s and 40 s). $6.8 \%$ of diabetics have an obesity problem with IMG being greater 
than $30.54 .6 \%$ of the diabetics had a IMG greater than 25. Carbohydrate intolerance in $5.3 \%$ of the population, when the evaluation was made, was within the worlwide accepted tolerance for glycemia of between $7.8 \mathrm{mmol}$ nad 11.1 $\mathrm{mmol}$ in blood.

$22.5 \%$ of diabetics had maternal diabetic antecedents and $9.8 \%$ paternal antecedents, making this statistically significant $(p<0.05) .11 .1 \%$ of the maternal grandfathers had diabetes mellitus. The most frequent obese family antecedent occurred in $26 \%$ of the mothers and in the maternal grandmothers; the same happened for $17 \%$ of the fathers and maternal grandfathers.

$44.7 \%$ of the identified diabetics were already known to be diabetic and $31.3 \%$ were already taking medication for their metabolical problems.

\section{REFERENCIAS}

1. Schmitt EW. Un nuevo concepto en la terapéutica de la diabetes mellitus. Inbición de las $\alpha$-glucosidasas por la acarbosa. 1988 BayerAG, Leverkusen. Mennen, Asten, Paises Bajos.

2. Jarrett JR. The epidemiological of diabetes mellitus. In: Pickup J, Williams G eds. Textbook of Diabetes Oxford. Balckwell Scientific Publications 1991;47:53.

3. Organizacion Mundial de la Salud. Diabetes mellitus. Informe de un grupo de estudio de la OMS. Serie de informes técnicos 727. OMS, Ginebra 1985.

4. Halter JB, Porte D. The clinical syndrome of diabetes mellitus. In: Dyck, Thomas PK, Asbury AT, Porte D. eds. Diabetes neuropathy. Philadelphia: WB Saunders 1987;3:26.

5. Heath and Public Policy Committee, American College of Physicians. Selected methods for the managenent of diabetes mellitus. Ann Intern Med 1983;99:272. Cit: Dyck, Thomas PK, Asbury AT, Porte D. eds. Diabetes neuropathy. Philadelphia: WB Saunders 1987;3:26.

6. Huggett A ST G, Nixon DA. Use of glucose oxidase, peroxidase and o-dianisidine in determination of blood and urinary glucose. Lancet 1957;2:368-370.

7. Niejadlik DC, Dube AH, Adamko SM. Glucose measurements and clinical correlations. JAMA 1973;224:1734-13736.

8. Everhart J, nowler WC, Bennett PH. Incidence and risk factors for noninsulin-dependent diabetes. In Harris
MI, amman RF, eds. Diabetes in America: Diabetes data compiled 1984. NIH Publication N 85-1468:4:1-35.

9. Diabetes Epidemiological Research International. Preventing insulin dependent diabetes mellitus. $\mathrm{Br}$ Med J 1987;295:479-481.

10. Pickup J, Williams Geds. Textbook of Diabetes Oxford. Balckwell Scientific Publications 1991.

11. Salvador AM, Pérez HM. Prevalencia de diabetes mellitus en la población adulta en un área de salud del municipio de Santiago de Cuba. Rev Cuba Hig Epidemiol $1987 ; 25(2) 205-213$.

12. Cortazar $\mathrm{J}$ eta al. La diabetes en Colombia. En: Curso Panamericano para Graduados "Diabetes Mellitus en Medicina General». Abril 1980, Bogotá, Harvard Medical School Departament of Counting Education Joslin Diabetes Foundation Inc.

13. Triana A, Cortés $S$, Hoyos $N$, López C, Remolina $E$, Rodríguez B. Encuesta de prevalencia de Diabetes en Fresno, Tolima. IV Congreso Latinoamericano de Diabetes, Caracas Venezuela. 1980.

14. López C, Cortés S, Hoyos N, Triana A, Castillo D, Sánchez Medina M. Encuesta de prevalencia de diabetes en individuos con alto riesgo en Fusagasuga, Cund. IV Congreso Latinoamericano de Diabetes, Caracas Venezuela, 1980;41.

15. Eisenbarth GS. Type / diabetes mellitus: a chronic autoinmune disease. NEngl J Med 1986;314:1360-1368.

16. Ferrannini $E$, DeFronzo RA. The association of hypertension, diabetes, and obesity: a revieww. J Nephrol 1989:1:3-15.

17. Reaven GM. Role of insulin resistance in human disease. Diabetes 1988;37:1595-606

18. Burditt AGF, Caird FI, Draper GJ. The natural history of diabetic retinopathy. Q J Med 1968;37:303-317.

19. Nathan DM, Singer $D E$, Godine JE, Harrington $\mathrm{CH}$, Perimuterm LC. Rethinopathy in older type II diabetics: associations with glucose control. Diabetes 1986; 35:797-801.

20. Forrest RD, Jackson CA, Yudkin JS. The glycohaemoglobin assay as a screening test for diabetes mellitus: the Islington Diabetes Survey. Diabetic Med $1987 ; 4: 254-259$

21. Cederholm J, Ronquist G, Wibell L. Comparison of glycosylated hemoglobin with the oral glucose tolerance test. Diabete Metab 1984;10:224-229.

22. Bolli $G$, Compagnucci $P$, Cartechini $M G$ et al. $\mathrm{HbA1}$ in sujets with abnormal glucose tolerance but normal fasting plasma glucose. Diabetes 1980;29-272-277. 
23. Assmann $G$, Lewis $B$, Mancini $M$, Stein $Y$ et al. Diagnóstico y tratamiento de la hiperlipidemia en adultos. Una normativa de la European Atherosclerosis Society (EAS) Valencia, 24 Octubre 1987. En: Assmann G. Carmena R (ED) Trastornos del metabolismo de los lípidos y cardiopatía coronaria. Prevención primaria, diagnóstico y directrices terapéuticas para la práctica clinica. Munchen: Editorial MMV, 1990,p 38-98.
24. Forrest RD, Jackson CA, Yudkin JS. The glycohaemoglobin assay as a screening test for diabetes mellitus: the Islington Diabetes Survey. Diabetic Med 1987:4:254-259.

25. Cederholm J, Ronquist G, Wibell L. Comparison of glycosylated hemoglobin with the oral glucose tolerance test. Diabete Metab 1984:10:224-229. 